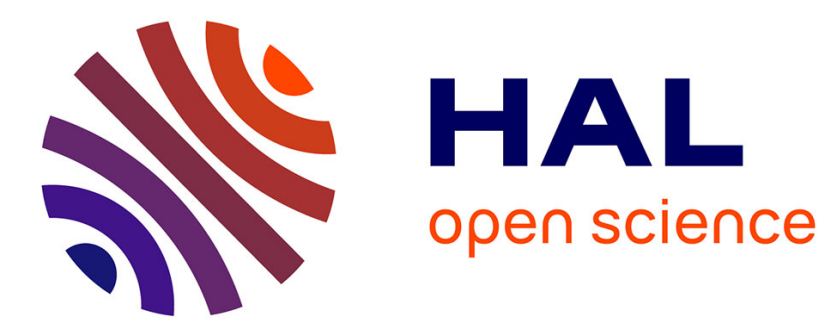

\title{
The m-Bézout Bound and Distance Geometry
}

Evangelos Bartzos, Ioannis Z. Emiris, Charalampos Tzamos

\section{To cite this version:}

Evangelos Bartzos, Ioannis Z. Emiris, Charalampos Tzamos. The m-Bézout Bound and Distance Geometry. CASC 2021 - 23rd International Workshop Computer Algebra in Scientific Computing, Sep 2021, Sochi, Russia. 10.1007/978-3-030-85165-1_2 . hal-03523833

\section{HAL Id: hal-03523833 https://hal.science/hal-03523833}

Submitted on 12 Jan 2022

HAL is a multi-disciplinary open access archive for the deposit and dissemination of scientific research documents, whether they are published or not. The documents may come from teaching and research institutions in France or abroad, or from public or private research centers.
L'archive ouverte pluridisciplinaire HAL, est destinée au dépôt et à la diffusion de documents scientifiques de niveau recherche, publiés ou non, émanant des établissements d'enseignement et de recherche français ou étrangers, des laboratoires publics ou privés. 


\section{Metadata of the chapter that will be visualized in SpringerLink}

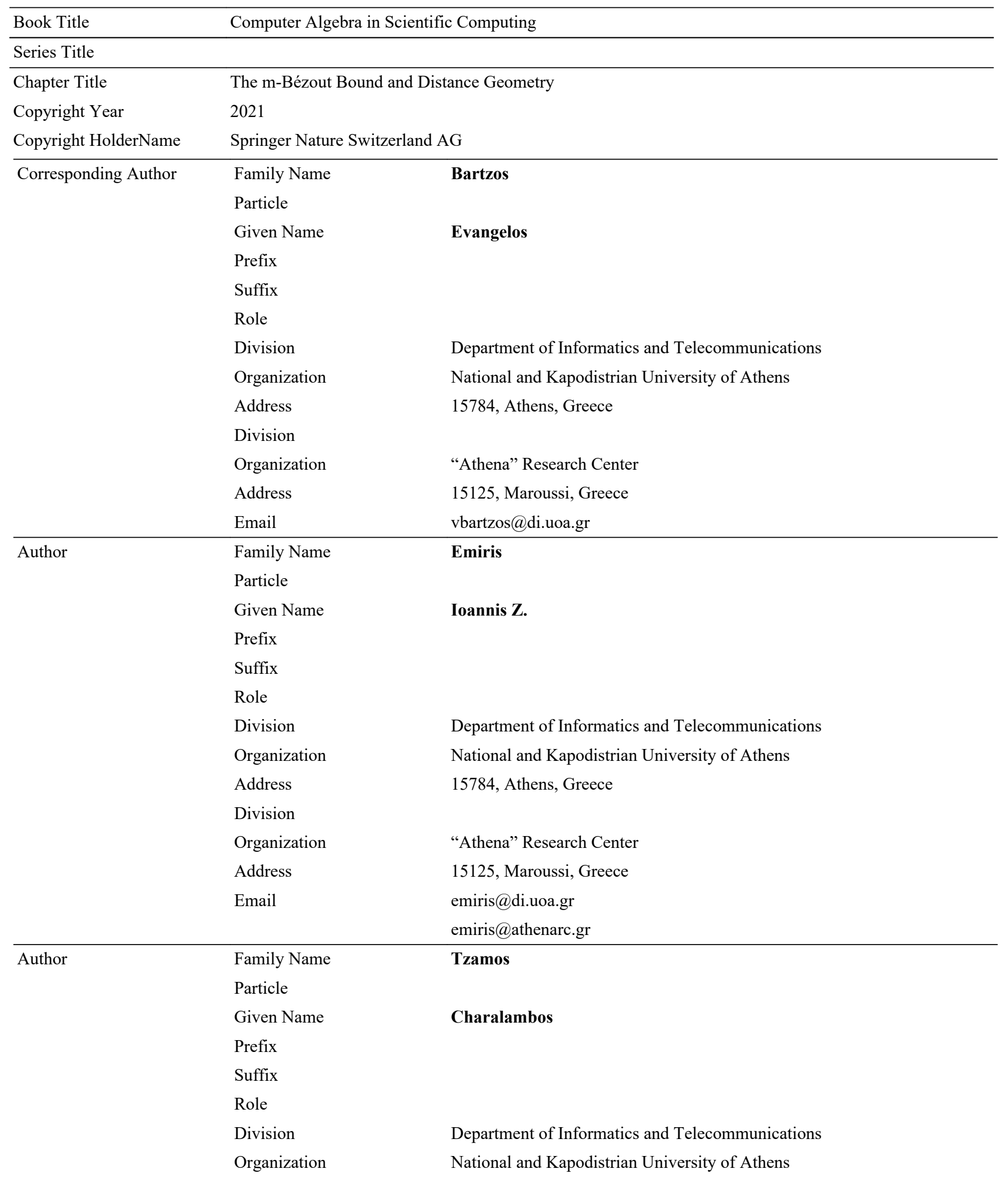


We offer a closed form bound on the m-Bézout bound for multi-homogeneous systems whose equations include two variable subsets of the same degree. Our bound is expectedly not tight, since computation of the $\mathrm{m}$-Bézout number is \#P-hard by reduction to the permanent. On the upside, our bound is tighter than the existing closed-form bound derived from the permanent, which applies only to systems characterized by further structure.

Our work is inspired by the application of the m-Bézout bound to counting Euclidean embeddings of distance graphs. Distance geometry and rigidity theory study graphs with a finite number of configurations, up to rigid transformations, which are prescribed by the edge lengths. Counting embeddings is an algebraic question once one constructs a system whose solutions correspond to the different embeddings.

Surprisingly, the best asymptotic bound on the number of embeddings had for decades been Bézout's, applied to the obvious system of quadratic equations expressing the length constraints. This is essentially $2^{d n}$, for graphs of $n$ vertices in $d$ dimensions, and implies a bound of $4^{n}$ for the most famous case of Laman graphs in the plane. However, the best lower bound is about $2.5^{n}$, which follows by numerically solving appropriate instances.

In [3], the authors leverage the m-Bézout bound and express it by the number of certain constrained orientations of simple graphs. A combinatorial process on these graphs has recently improved the bound on orientations and, therefore, has improved the bounds on the number of distance graph embeddings [4]. For Laman graphs the new bound is inferior to $3.8^{n}$ thus improving upon Bézout's bound for the first time. In this paper, we obtain a closed-form bound on the m-Bézout number of a class of multi-homogeneous systems that subsumes the systems encountered in distance graph embeddings. 


\title{
The m-Bézout Bound and Distance Geometry
}

\author{
Evangelos Bartzos $^{1,2(\bowtie)}$, Ioannis Z. Emiris ${ }^{1,2}$, and Charalambos Tzamos ${ }^{1}$ \\ 1 Department of Informatics and Telecommunications, \\ National and Kapodistrian University of Athens, 15784 Athens, Greece \\ \{vbartzos, emiris, ctzamos\}@di.uoa.gr \\ 2 "Athena" Research Center, 15125 Maroussi, Greece \\ emiris@athenarc.gr
}

\begin{abstract}
We offer a closed form bound on the m-Bézout bound for multi-homogeneous systems whose equations include two variable subsets of the same degree. Our bound is expectedly not tight, since computation of the m-Bézout number is \#P-hard by reduction to the permanent. On the upside, our bound is tighter than the existing closed-form bound derived from the permanent, which applies only to systems characterized by further structure.

Our work is inspired by the application of the m-Bézout bound to counting Euclidean embeddings of distance graphs. Distance geometry and rigidity theory study graphs with a finite number of configurations, up to rigid transformations, which are prescribed by the edge lengths. Counting embeddings is an algebraic question once one constructs a system whose solutions correspond to the different embeddings. Surprisingly, the best asymptotic bound on the number of embeddings had for decades been Bézout's, applied to the obvious system of quadratic equations expressing the length constraints. This is essentially $2^{d n}$, for graphs of $n$ vertices in $d$ dimensions, and implies a bound of $4^{n}$ for the most famous case of Laman graphs in the plane. However, the best lower bound is about $2.5^{n}$, which follows by numerically solving appropriate instances.

In [3], the authors leverage the m-Bézout bound and express it by the number of certain constrained orientations of simple graphs. A combinatorial process on these graphs has recently improved the bound on orientations and, therefore, has improved the bounds on the number of distance graph embeddings [4]. For Laman graphs the new bound is inferior to $3.8^{n}$ thus improving upon Bézout's bound for the first time. In this paper, we obtain a closed-form bound on the m-Bézout number of a class of multihomogeneous systems that subsumes the systems encountered in distance graph embeddings.
\end{abstract}

Keywords: Graph embeddings - Graph orientations · Multihomogeneous Bézout bound · Matrix permanent

EB was fully supported by project ARCADES which has received funding from the European Union's Horizon 2020 research and innovation programme under the Marie Skłodowska-Curie grant agreement No 675789. The authors are members of team AROMATH, joint between INRIA Sophia-Antipolis, France, and NKUA.

(C) Springer Nature Switzerland AG 2021

F. Boulier et al. (Eds.): CASC 2021, LNCS 12865, pp. 1-15, 2021.

https://doi.org/10.1007/978-3-030-85165-1_2 


\section{Introduction}

Distance Geometry is the branch of mathematics studying configurations of sets of points, when only (some of) their distances are known. Given a set of distances for some pairs of points, one of the main problems in Distance Geometry is to determine the unspecified distances. This is highly motivated by applications in molecular biology [16], robotics [26], civil engineering [1,13], sensor network localization [27], data science [18], material theory [8,22].

Rigidity theory studies the properties of graphs that have rigid embeddings in Euclidean space for fixed edge weights that represent length between points. Rigidity is defined for a specific embedding space. Let $G=(V, E)$ be a simple undirected graph and $\mathbf{p}=\left\{p_{1}, \ldots, p_{|V|}\right\} \in \mathbb{R}^{d \cdot|V|}$ be a conformation of $|V|$ points in $\mathbb{R}^{d}$. The framework $G(\boldsymbol{p})$ is rigid if and only if there are only finite embeddings that satisfy the given edge lengths $\boldsymbol{\lambda}=\left(\left\|p_{u}-p_{v}\right\|\right)_{(u, v) \in E}$ induced by $\boldsymbol{p}$, where $p_{v} \in \mathbb{R}^{d}$ are the coordinates of vertex $v$. A graph is generically rigid if it is rigid for almost all conformations and this is a property of the underlying graph (and not of the specific embedding). In other words, genericity refers to the prescribed edge lengths of the graph.

A major open problem in rigidity theory is to find tight upper bounds on the number of realizations of minimally rigid graphs, e.g. [15]; we refer to this number as embedding number. A Euclidean embedding is related to the real solutions of a well-constrained system of algebraic equations. The complex solutions extend the notion of real to complex embeddings and allow one to leverage complex algebraic geometry. Direct application of Bézout's bound of the quadratic polynomial system that corresponds to the edge constraints yields a bound of $\mathcal{O}\left(2^{d \cdot|V|}\right)$. In [7], they presented an upper bound that had been the best until recently, applying a theorem on the degree of determinantal varieties [14]. However, it does not improve asymptotically upon Bézout's. For $d=2$, techniques using mixed volume have been introduced in [24], without managing to improve the bound. A recent result in algebraic frame theory establishes a bound on the degree of the projections of finite unit norm tight frames [5] using algebraic matroids.

Two recent publications dealing with that problem managed to improve the asymptotic bound based on the combinatorial properties of minimally rigid graphs. This is the approach on which the present work relies. In [3], outdegreeconstrained orientations as well as matrix permanents are related to the mBézout bound of certain algebraic systems that compute the embedding number. This work resulted to improved asymptotic upper bounds for $d \geq 5$, using the Brégman-Minc permanent bound [9,21]. More importantly, this work led to the following combinatorial technique. In [4], the target is on a method that bounds the number of outdegree-constrained orientations. It managed to improve the bound on embeddings for all $d \geq 2$ (the case of $d=1$ is trivial) and proved that the permanent bounds can be ameliorated in that case. For instance, in the case of $d=2$, this approach results to an upper bound of $\mathcal{O}\left(3.77^{n}\right)$, while the Bézout bound is $\mathcal{O}\left(4^{n}\right)$. 
It is well known that, applied to the same system, Bézout's bound is smaller or equal to the multi-homogeneous Bézout bound (m-Bézout) [23], which is smaller or equal to the BKK bound expressed by mixed volume [6]. The bounds coincide for dense systems, where all coefficients for a given total degree are nonzero, but differ as the system becomes sparser. Of course, each bound counts roots in a different ambient variety. These bounds are compared in [12], with emphasis on computing mixed volume, which coincides with the m-Bézout number for multihomogeneous systems whose maximal monomials have nonzero coefficients. Formally, the latter condition requires that none of the monomials corresponding to vertices of the Newton polytopes vanishes.

Computing the m-Bézout number for a given variable partition is \#P-hard by reduction to the permanent, which is the cornerstone \#P-hard problem. The same hardness result holds for mixed volume, which coincides with the m-Bézout number for certain polynomial structures; when the system is sparse, in order words has certain zero coefficients, the mixed volume may be smaller. Moreover, it is known that mixed volume is APX-hard, in other words it is hard to deterministically approximate it within an error which is asymptotically smaller than exponential in the system's number of variables. Another problem is, given an algebraic system, to find the optimal variable partition so that the system is modeled as a multi-homogeneous one with minimum m-Bézout number, see Definition 2. This problem is not in APX, unless $\mathrm{P}=\mathrm{NP}$ [19].

Recently, other approaches came to our attention relating polynomial systems with graph theoretical concepts. More precisely, there are connections of the polynomial system with chordal graphs in order to enhance Cylindrical Algebraic Decomposition (CAD) [17] and Gröbner bases [10] algorithms.

Our Contribution. In this paper, we generalize the aforementioned approach to bounding the m-Bézout bound of a quite general class of multi-homogeneous polynomial systems, which subsumes the class of systems encountered in rigidity theory. We exploit the connection between the system's m-Bézout number and the number of constrained orientations of a simple graph that we specify for the systems under investigation, then bound the number of the graph's orientations. This procedure relies on the proofs in $[3,4]$. It offers the first closed-form bound on m-Bézout numbers; we hope this may prove useful in a fast estimation of the algebraic complexity of problems modeled by multi-homogeneous algebraic equations. Trivially, our closed form upper bounds the mixed volume of these multi-homogeneous systems.

Our main result concerns any multi-homogeneous 0-dimensional polynomial system $\boldsymbol{P}(\boldsymbol{x})=\left(P_{1}(\boldsymbol{x}), P_{2}(\boldsymbol{x}), \ldots, P_{m}(\boldsymbol{x})\right)$ that cannot be split to smaller subsystems: formally, there is no subset of equations $\boldsymbol{P}^{\prime}$ including only a subset of variables that do not appear in $\boldsymbol{P} \backslash \boldsymbol{P}^{\prime}$. The multi-homogeneous structure is manifest by partitioning the variables to subsets $\left(X_{1}, X_{2}, \ldots X_{n}\right)$ with $\left|X_{i}\right|=d_{i}, d_{1}+\cdots+d_{n}=m$, so that each $P_{i}$ is homogeneous in each $X_{j}$ (see Definition 2 for more details). 
Theorem 1. Given multi-homogeneous system $\boldsymbol{P}$ as above, let us assume that

- every $P_{i}$ contains at most two variable subsets,

- two polynomials $P_{i}, P_{j}$ do not contain the same pair of variables, and

- the degree of each $P_{i}$, denoted by $\delta_{i}$, is the same in both variable sets.

Let $d=\max _{1 \leq i \leq n}\left(d_{i}\right), k=n d-m$, then the m-Bézout number of $\boldsymbol{P}$ is bounded by

$$
\alpha_{d}^{n} \cdot \beta_{d}^{k-1} \cdot \prod_{i=1}^{m} \delta_{i}
$$

where

$$
\alpha_{d}=\max _{p \geq d}\left(2^{p-d}\left(\begin{array}{l}
p \\
d
\end{array}\right)^{2 d-3}\right)^{\frac{1}{2 p-3}}, \beta_{d}=\left(2\left(\begin{array}{l}
p \\
d
\end{array}\right)^{-2}\right)^{\frac{1}{2 p-3}},
$$

and $p \in \mathbb{N}$ appearing in $\beta_{d}$ is the one which maximizes $\alpha_{d}$.

Notice that $\beta_{d}<1$, so $a_{d}^{n}$ gives the asymptotic order of this bound. An asymptotic expression of $a_{d}$ is given in [4]:

$$
\alpha_{d} \simeq \sqrt{\frac{1}{2}\left(\begin{array}{c}
2 d \\
d
\end{array}\right)}\left(1+\mathcal{O}\left(\frac{\ln ^{2} d}{d}\right)\right) .
$$

Upper bounds on $\alpha_{d}^{n}$ are provided in Table 1 .

Table 1. Upper bounds on $\alpha_{d}^{n}$

\begin{tabular}{l|l|l|l|l|l|l|l|l}
\hline$d$ & 2 & 3 & 4 & 5 & 6 & 7 & 8 & 9 \\
\hline$\alpha_{d}^{n}$ & $1.88^{n}$ & $3.41^{n}$ & $6.34^{n}$ & $11.9^{n}$ & $22.7^{n}$ & $43.7^{n}$ & $84.4^{n}$ & $163.7^{n}$ \\
\hline
\end{tabular}

Paper Structure. The rest of the paper is organized as follows. In Sect.2, we discuss established methods that relate the m-Bézout bound with the number of orientations of a graph, and methods that improve the upper bounds on the number of embeddings. In Sect.3, we extend these methods to a class of multihomogeneous systems, thus bounding their m-Bézout number. Finally, in Sect. 4 we present concluding remarks and present ideas of future work.

\section{Bounds on the Embedding Number}

In this section, we start by offering further background on rigid graphs. Then we present previous work, that relates the number of orientations of a graph to the m-Bézout, and methods that harness this relation to improve the asymptotic upper bounds on the embedding number. 
A generically minimally rigid graph is a rigid graph that loses the rigidity property if any of its edges is removed. A fundamental theorem in graph rigidity due to Maxwell, gives a necessary condition for a graph and all its subgraphs to be rigid. In particular, if a graph $G$ is minimally rigid in $\mathbb{R}^{d}$, then $|E|=d \cdot|V|-\left(\begin{array}{c}d+1 \\ 2\end{array}\right)$, and for every subgraph $G^{\prime}\left(V^{\prime}, E^{\prime}\right) \subset G$ it holds that $\left|E^{\prime}\right| \leq d \cdot\left|V^{\prime}\right|-\left(\begin{array}{c}d+1 \\ 2\end{array}\right)[20]$. Below this number of edge constraints shall become quite intuitive since it equals the number of unknown variables in the respective algebraic system.

In order to compute the embeddings of a rigid graph up to rigid motions, we use the following formulation used also in [11,24], which is called sphere equations in [2].

Definition 1 ([2]). Let $G=(V, E)$ be a graph. We denote by $\boldsymbol{\lambda}$ the lengths of the edges on $G$ and by $\tilde{X}_{u}=\left\{x_{u, 1}, \ldots, x_{u, d}\right\}$ the $d$ variables that correspond to the coordinates of a vertex $u$. The following system of equations gives the embedding number for $G$ :

$$
\begin{gathered}
\left\|\tilde{X}_{u}\right\|^{2}=s_{u}, \quad \forall u \in V \\
s_{u}+s_{v}-2\left\langle\tilde{X}_{u}, \tilde{X}_{v}\right\rangle=\lambda_{u, v}^{2}, \quad \forall(u, v) \in E \backslash E\left(K_{d}\right)
\end{gathered}
$$

where $\left\langle\tilde{X}_{u}, \tilde{X}_{v}\right\rangle$ is the Euclidean inner product. The first set of equations shall be called magnitude equations, while the second are the edge equations.

This formulation is suitable for sparse elimination theory (see [3] for a general discussion on the algebraic system). In order to factor out rigid motions, if $G$ possesses a complete subgraph in $d$ vertices, the coordinates of these vertices shall be fixed.

Notice that, when we fix $d$ vertices, the above algebraic system has $d \cdot n-d^{2}$ edge equations and $n-d$ magnitude equations. In [3] the variables are partitioned into subsets, such that each subset of variables contains these ones which correspond to the coordinates and the magnitude of a vertex $X_{u}=\tilde{X}_{u} \cup\left\{s_{u}\right\}$.

Let us formally define multi-homogeneous systems in general, thus subsuming the systems presented in the Introduction.

Definition 2. Let $\boldsymbol{x}$ be a vector of $m$ variables and $\boldsymbol{P}(\boldsymbol{x})$ be a system of $m$ polynomial equations in $\mathbb{C}[\boldsymbol{x}]$. Let $X_{1}=\left(x_{1,1}, x_{1,2}, \ldots, x_{1, d_{1}}\right), X_{2}=\left(x_{2,1}, x_{2,2}, \ldots\right.$, $\left.x_{2, d_{2}}\right), \ldots, X_{n}=\left(x_{n, 1}, x_{n, 2}, \ldots, x_{n, d_{n}}\right)$ be a partition of the affine variables, such that $\left|X_{i}\right|=d_{i}$, and $d_{1}+\cdots+d_{n}=m$. The degree of a polynomial $P_{i}$ in a variable set $X_{j}$ is the same as the degree of this polynomial, if all variables $x_{j^{\prime}, k} \notin X_{j}$ were treated as coefficients and is denoted with $\delta_{i, j}$. Every $P_{i}$ is homogeneous in each variable set $X_{j}$, with homogenizing variable $x_{i, 0}$ and multidegree specified by vector $\boldsymbol{\delta}_{\boldsymbol{i}}=\left(\delta_{i, 1}, \delta_{i, 2}, \ldots, \delta_{i, n}\right)$. Then $\boldsymbol{P}$ is multi-homogeneous of type

$$
\left(d_{1}, \ldots, d_{n} ; \boldsymbol{\delta}_{1}, \ldots, \boldsymbol{\delta}_{n}\right) .
$$

If all positive entries have the same value in a multidegree vector $\boldsymbol{\delta}_{\boldsymbol{i}}$, then this value will be denoted with $\operatorname{mdeg}\left(P_{i}\right)$. 
Let us recall a classic theorem from algebraic geometry, see e.g. [23], defining the m-Bézout bound.

Theorem 2. Consider the multi-homogeneous system $\boldsymbol{P}(\boldsymbol{x})$ defined above. The coefficient of the monomial $Y_{1}^{d_{1}} \cdots Y_{n}^{d_{n}}$ in the polynomial defined by the product

$$
\prod_{i=1}^{m}\left(\delta_{i, 1} \cdot Y_{1}+\cdots+\delta_{i, n} \cdot Y_{n}\right) \text {. }
$$

bounds the number of roots of $\boldsymbol{P}(\boldsymbol{x})$ in $\mathbb{P}^{d_{1}} \times \cdots \times \mathbb{P}^{d_{n}}$, where $Y_{i}$ are new symbolic parameters, and $\mathbb{P}^{j}$ is the $j$-dimensional projective space over $\mathbb{C}$. The bound is tight for generic coefficients of $\boldsymbol{P}(\boldsymbol{x})$.

The most efficient method to compute the m-Bézout bound is by evaluating the permanent of a matrix capturing the polynomial structure, see [12]. Let this matrix be $A$ for a multi-homogeneous system $\boldsymbol{P}$ as above, and let $\operatorname{per}(A)$ denote the permanent of this matrix. Then the m-Bézout bound equals

$$
\frac{1}{d_{1} ! d_{2} ! \cdots d_{n} !} \cdot \operatorname{per}(A)
$$

By applying Theorem 2, the following expansion is considered in the case of sphere equations (see Definition 1):

$$
\prod_{u \in V^{\prime}} 2 \cdot Y_{u} \prod_{(u, v) \in E^{\prime}}\left(Y_{u}+Y_{v}\right)=2^{n-d} \cdot \prod_{u \in V^{\prime}} Y_{u} \prod_{(u, v) \in E^{\prime}}\left(Y_{u}+Y_{v}\right)
$$

where $G^{\prime}\left(V^{\prime}, E^{\prime}\right)=G \backslash K_{d}$. Thus, it suffices to find the coefficient of $\prod_{u \in V^{\prime}} Y_{u}^{d}$ in the expansion of the product:

$$
\prod_{(u, v) \in E^{\prime}}\left(Y_{u}+Y_{v}\right)
$$

In [3], it is proven that this coefficient equals the cardinality of the set of those orientations of $G^{\prime}=\left(V, E \backslash E\left(K_{d}\right)\right)$ satisfying the conditions set in the following theorem.

Theorem 3 ([3]). Let $G=(V, E)$ be a minimally rigid graph that contains at least one complete subgraph on $d$ vertices, denoted by $K_{d}=\left(v_{1}, \ldots, v_{d}\right)$. Let $\mathcal{B}\left(G, K_{d}\right)$, stand for the number of outdegree-constrained orientations of $G^{\prime}=$ $\left(V, E \backslash E\left(K_{d}\right)\right)$, such that:

- the outdegree of $v_{1}, \ldots, v_{d}$ is 0 .

- the outdegree of every vertex in $V \backslash\left\{v_{1}, \ldots, v_{d}\right\}$ is $d$.

The orientations that satisfy these constraints are called valid. Then the number of embeddings of $G$ in $\mathbb{C}^{d}$, does not exceed

$$
2^{|V|-d} \cdot \mathcal{B}\left(G, K_{d}\right)
$$



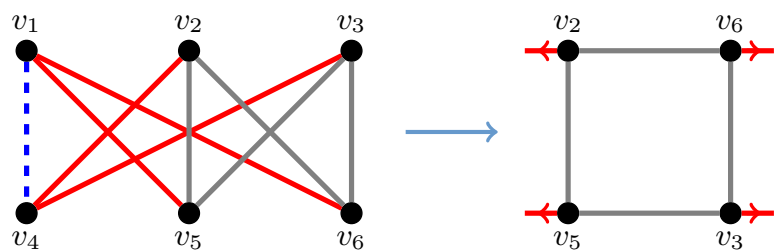

Fig. 1. Left: Graph $K_{3,3}$ where $v_{1}, v_{2}$ are chosen as fixed vertices $(d=2)$. Right: the resulting pseudograph, after removing the fixed vertices.

The theorem extends to the case where a fixed $K_{d}$ does not exist [4].

In [4], this method yields the current record upper bounds on the number of embeddings. To achieve this, the valid orientations of Theorem 3 are associated to a graphical structure in which the vertices that have fixed outdegree 0 are omitted. This graphical structure is called pseudograph [4], and extends the notion of a standard graph by allowing hanging edges, which have a single endpoint; hanging edges are always oriented outwards from its incident vertex. In correspondence with Theorem 3, the hanging edges represent edges incident to the missing vertices in the original graph. It is thus a collection $\mathcal{G}=(V, E, H)$, where $V$ denotes the vertices, $E$ the edges with two endpoints and $H$ the hanging edges.

An elimination process that applies to a pseudograph bounds the number of orientations. At each step, one or more vertices (see Fig. 2) are removed from the pseudograph and their incident edges are either removed or become hanging edges in a smaller graph. The number of possible outcomes in every step multiplies the current count until a terminal condition is reached; the overall product bounds the number of valid orientations.

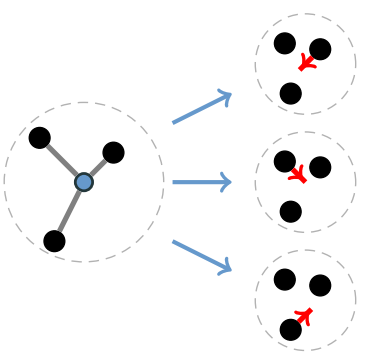

Fig. 2. Excerpt from [4]. Left: a (blue) vertex with 3 neighbours and no hanging edges. Right: 3 possible cases for the orientation, after the removal of the blue vertex, when $d=2$. The number of possible cases is multiplied in every elimination step, which eventually bounds the number of valid orientations. (Color figure online) 
We remark that from an algebraic point of view, the hanging edges correspond to variables that can be eliminated linearly using the edge equations from Definition 1. In other words, they represent a reduction in the cardinality of the variables set of the specific vertex (see Fig. 1).

Theorem 4 ([4]). Let $B_{d}(n, k)$ denote the maximal number of orientations with outdegree $d$ for a connected pseudograph with $n$ vertices and $k$ hanging edges. Then it holds that:

$$
B_{d}(n, k) \leq \alpha_{d}^{n} \cdot \beta_{d}^{k-1}
$$

where $\alpha_{d}$ and $\beta_{d}$ are defined as in Theorem 1.

For $d=2, \ldots, 9$, the formula yields improved bounds on the number of orientations which are expressed by $a_{d}^{n}$, see Table 1 , since $\beta_{d}<1$. Due to Theorem 3 , these quantities multiplied by $2^{n}$, bound the number of embeddings in the $d$ dimensional complex space. In the case of $d=2$ and $d=3$, this improved the asymptotic bound on the embedding number to $\mathcal{O}\left(3.77^{n}\right)$ and $\mathcal{O}\left(6.82^{n}\right)$ respectively.

\section{Algebraic Systems Modeled by Simple Graphs}

In this section we exploit the methods described above to bound the m-Bézout number of a class of multi-homogeneous algebraic systems that shall be modeled via a simple graph.

Recall the polynomial systems described in Theorem 1: For every polynomial $P_{i}$ containing variable sets $X_{u}, X_{v}$, it holds for the degree $\operatorname{mdeg}\left(P_{i}\right)=\delta_{i, j}$ only for $j \in\{u, v\}$, whereas $\delta_{i, j}=0$, for all $j \notin\{u, v\}$. We also require that the polynomial system cannot be split into smaller subsystems with disjoint variables, and that two different polynomials cannot contain the same pair of variable sets.

We call such systems simple graph polynomial systems since they define a simple connected graph $G(\boldsymbol{P})=(V, E)$ as follows: The vertices of $G$ correspond to the $n$ variable subsets, while each polynomial yields an edge whose endpoints are the respective vertices. There are no loops, because no polynomial contains a single variable set. Since the pair of variable sets is unique for each polynomial, there can be only one edge with the same endpoints, hence no multiple edges appear. Furthermore, if the graph was disconnected, every connected component would contain vertices corresponding to sets of variables that do not appear in the other connected components, which has been excluded. All these conditions indicate that the graph is simple and connected.

The main observation here is that we can relate the m-Bézout bound in the cases of simple graph polynomial systems with valid orientations, as described in Sect. 2, but we can relax those conditions since it is not necessary to restrain these constraints to outdegree $d$ and outdegree 0 cases (see Theorem 3 ). 
Theorem 5. Let $\boldsymbol{P}$ be a simple graph polynomial system with $m$ equations for a partition of variables $X_{1}, X_{2}, \ldots, X_{n}$ and let $G(\boldsymbol{P})=(V, E)$ be the associated simple graph. Let $\left|X_{j}\right|=d_{j}, \boldsymbol{d}=\left(d_{1}, d_{2}, \ldots d_{n}\right)$ and $\operatorname{mdeg}\left(P_{(u, v)}\right)=\delta_{(u, v)}$, where $(u, v)$ is the edge associated with the polynomial containing $X_{u}, X_{v}$. We denote by $\mathcal{B}(G(\boldsymbol{P}), \boldsymbol{d})$ the number of orientations of $G(\boldsymbol{P})$, constrained so that each vertex $u$ representing $X_{u}$ has outdegree $d_{u}$. Then, the $m$-Bézout number for $\boldsymbol{P}$ under this variable partition is exactly

$$
\mathcal{B}(G(\boldsymbol{P}), \boldsymbol{d}) \cdot \prod_{(u, v) \in E} \delta_{(u, v)} .
$$

Proof. The m-Bézout bound is the coefficient of the term $\boldsymbol{Y}=Y_{1}^{d_{1}} \cdots Y_{2}^{d_{2}} \cdot Y_{n}^{d_{n}}$ in the polynomial $\prod_{(u, v) \in E}\left(\delta_{(u, v)} \cdot Y_{u}+\delta_{(u, v)} \cdot Y_{v}\right)$, where every $Y_{k}$ is a new symbolic parameter. Clearly the latter is equal with

$$
\left(\prod_{(u, v) \in E} \delta_{(u, v)}\right) \cdot \prod_{(u, v) \in E}\left(Y_{u}+Y_{v}\right) .
$$

Using a similar argument to that in the proof of Theorem 3 in [3], the monomial $\boldsymbol{Y}$ appears only if each term $Y_{u}$ is selected exactly $d_{u}$ times in the expansion of this product. Since each set of variables represents a vertex and each polynomial represents an edge in $G(\boldsymbol{P})$, this can be connected to $d_{u}$ edges directed outwards from $u$ in a graph orientation.

Now, we can derive general upper bounds on the m-Bézout number using the pseudograph formulation. Combining Theorem 5 and Theorem 4 leads to the following proof of Theorem 1.

Proof (of Theorem 1). Let $d=\max (\boldsymbol{d})$, for a system $\boldsymbol{P}$, with $\boldsymbol{d}$ as defined above. Let $\mathcal{G}=(V, E, H)$ be a pseudograph, such that $V, E$ are the vertices and the edges of $G(\boldsymbol{P})$, respectively, $H$ are the hanging edges, where a vertex $v$ has exactly $d-d_{v}$ hanging edges as specified in Sect. 2 . Now, if a vertex $v$ has no hanging edges, then all of its $d_{v}=d$ edges should be directed outwards from it. On the other hand, for a vertex $v$ that has $k_{v}=d-d_{v}$ hanging edges, then $d_{v}$ edges in $E$ should be out-directed, which correspond to $d_{v}$ edges directed outwards in $G(\boldsymbol{P})$. These cases capture exactly all valid orientations of $G(\boldsymbol{P})$. The latter orientations are used to compute the m-Bézout bound of a simple graph polynomial in Theorem 5 .

Now, it suffices to bound the number of valid orientations of this pseudograph, by extending the techniques of [4]. The bound on valid orientations with fixed outdegree $d$ for all pseudographs with $|V|=n$ vertices and $|H|=k$ hanging edges is given by Theorem 4, thus establishing that Equation (1) bounds the m-Bézout bound.

Let us present two examples of simple graph polynomial systems, by computing the m-Bézout number, and by deriving the bound in Theorem 1 that concerns all systems whose graph has the same vertices and hanging edges. 

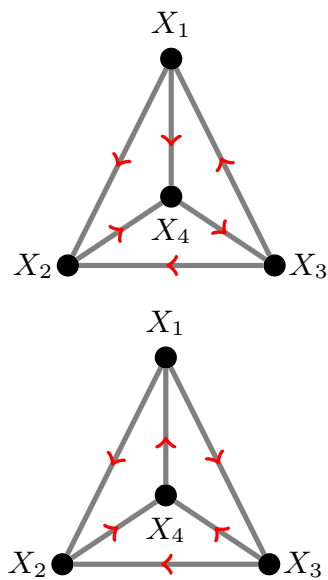
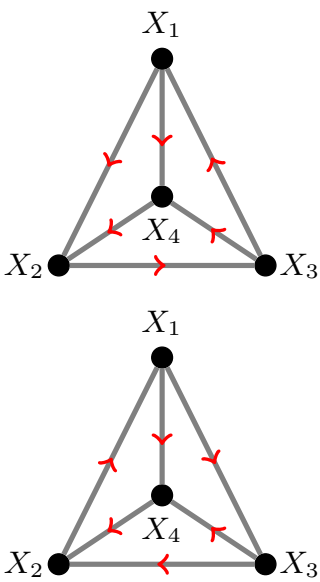

Fig. 3. The 4 outdegree-constrained orientations of $G(\boldsymbol{P})$ in Example 1. Since $\left|X_{1}\right|=$ $\left|X_{3}\right|=2,\left|X_{2}\right|=\left|X_{4}\right|=1$, the outdegree of $X_{1}, X_{3}$ is 2 , while that of $X_{2}, X_{4}$ is 1 .

Example 1. The following system $\boldsymbol{P}$ is a simple graph polynomial system:

$$
\begin{aligned}
& P_{\left(X_{1}, X_{2}\right)}=x_{1,1} x_{2,1}+5 x_{1,2} x_{2,1}+2 x_{1,2}+3 \\
& P_{\left(X_{1}, X_{3}\right)}=2 x_{1,1}^{2} x_{3,1}^{2}+2 x_{1,1}^{2} x_{3,2}^{2}+2 x_{1,2}^{2} x_{3,2}^{2}+x_{1,1} x_{1,2} x_{3,1} x_{3,2}+2 x_{1,2}-13 \\
& P_{\left(X_{1}, X_{4}\right)}=x_{1,1} x_{1,2} x_{4,1}^{2}-x_{1,1}^{2} x_{4,1}^{2}+x_{1,2} x_{4,1} \\
& P_{\left(X_{2}, X_{3}\right)}=4 x_{2,1}^{3} x_{3,1}^{2} x_{3,2}+x_{2,1} x_{3,1}^{2} x_{3,2}+2 x_{3,2}+7 \\
& P_{\left(X_{2}, X_{4}\right)}=2 x_{2,1} x_{4,1}+3 x_{2,1}+5 x_{4,1}-9 \\
& P_{\left(X_{3}, X_{4}\right)}=4 x_{3,1} x_{4,1}+5 x_{3,2} x_{4,1}+7 x_{3,1}+2 x_{4,1}
\end{aligned}
$$

for the partition of variables $X_{1}=\left\{x_{1,1}, x_{1,2}\right\}, X_{2}=\left\{x_{2,1}\right\}, X_{3}=\left\{x_{3,1}, x_{3,2}\right\}$, $X_{4}=\left\{x_{4,1}\right\}$. Of course, it is sparse in the sense that not all expected terms appear with nonzero coefficient; hence, one would expect its mixed volume to be inferior to its $m$-Bézout number. The vertices of $G(\boldsymbol{P})$ are labeled by these subsets; the cardinalities are $\left|X_{1}\right|=\left|X_{3}\right|=2$ and $\left|X_{2}\right|=\left|X_{4}\right|=1$, hence $\boldsymbol{d}=(2,1,2,1)$. The edge set is:

$$
E=\left\{\left(X_{1}, X_{2}\right),\left(X_{1}, X_{3}\right),\left(X_{1}, X_{4}\right),\left(X_{2}, X_{3}\right),\left(X_{2}, X_{4}\right),\left(X_{3}, X_{4}\right)\right\}
$$

The multi-homogeneous degrees are $\delta_{\left(X_{1}, X_{2}\right)}=1, \delta_{\left(X_{1}, X_{3}\right)}=2, \delta_{\left(X_{1}, X_{4}\right)}=2$, $\delta_{\left(X_{2}, X_{3}\right)}=3, \delta_{\left(X_{2}, X_{4}\right)}=1, \delta_{\left(X_{3}, X_{4}\right)}=1$.

We compute the $m$-Bézout bound by Theorem 5. Since $d_{1}=d_{3}=2, d_{2}=$ $d_{4}=1$ the outdegree of vertices $X_{1}, X_{3}$ should be 2 , while that of $X_{2}, X_{4}$ should be 1 for a valid orientation. There are 4 such orientations (Fig. 3). Therefore the $m$-Bézout bound is $12 \cdot 4=48$. The BKK bound gives a tighter bound by exploiting sparseness: using phcpy [25], we found a mixed volume of 44, which is the actual number of complex roots. 
In order to apply Theorem 1, we set $d=\max (\boldsymbol{d})=2$, so $\alpha_{2}=24^{1 / 5}$ and $\beta_{2}=18^{-1 / 5}$. Since the number of vertices of $G(\boldsymbol{P})$ is $n=4$ and the number of equations $m=6$, we have $k=n d-m=2$, and $\prod_{E} \delta_{\left(X_{i}, X_{j}\right)}=12$, then the bound is $\left\lfloor 12 \cdot 24^{4 / 5} \cdot 18^{-1 / 5}\right\rfloor=85$.

Let us compare this estimate to the Bézout bound. The total degrees of the equations are 2, 4,6,2, 4, 2; the Bézout bound is therefore 768 .

In the second example the multidegree vector has either zeros or ones. This means that we can relate the m-Bézout bound to the permanent of a $(0,1)$ matrix $A$ capturing the polynomial structure. For this kind of matrices, there is a permanent bound, better known as the Brègman-Minc bound [9,21]. Therefore, we shall also compare this bound to ours.

Example 2. The following system $\boldsymbol{Q}$ is a simple graph polynomial system:

$$
\begin{aligned}
& Q_{\left(X_{1}, X_{2}\right)}=x_{1,1} x_{2,1}+2 x_{1,1}+3 x_{2,1} \\
& Q_{\left(X_{1}, X_{3}\right)}=2 x_{1,1} x_{3,1}+x_{1,1} x_{3,2}+x_{3,1}+x_{3,2}+2 x_{1,1} \\
& Q_{\left(X_{1}, X_{5}\right)}=5 x_{1,1} x_{5,1}+2 x_{1,1} x_{5,2}+x_{5,1}+x_{5,2}+x_{1,1} \\
& Q_{\left(X_{2}, X_{4}\right)}=9 x_{2,1} x_{4,1}+x_{2,1} x_{4,2}+x_{4,1}+x_{4,2}+x_{2,1} \\
& Q_{\left(X_{2}, X_{5}\right)}=9 x_{2,1} x_{5,1}+x_{2,1} x_{5,2}+x_{5,1}+x_{5,2}+x_{2,1} \\
& Q_{\left(X_{3}, X_{4}\right)}=4 x_{3,2} x_{4,1}+2 x_{3,2} x_{4,2}+5 x_{3,1} x_{4,1}+9 x_{3,1} x_{4,2}+x_{3,1}+x_{3,2}+x_{4,1} \\
& Q_{\left(X_{3}, X_{5}\right)}=3 x_{3,2} x_{5,1}+4 x_{3,2} x_{5,2}+x_{3,1} x_{5,1}+7 x_{3,1} x_{5,2}+x_{3,1}+x_{3,2}+2 x_{5,1} \\
& Q_{\left(X_{4}, X_{5}\right)}=x_{4,2} x_{5,1}+9 x_{4,2} x_{5,2}+3 x_{4,1} x_{5,1}+4 x_{4,1} x_{5,2}+2 x_{4,1}+x_{4,2}+14 x_{5,1}
\end{aligned}
$$

for the partition of variables $X_{1}=\left\{x_{1,1}\right\}, X_{2}=\left\{x_{2,1}\right\}, X_{3}=\left\{x_{3,1}, x_{3,2}\right\}$, $X_{4}=\left\{x_{4,1}, x_{4,2}\right\}, X_{5}=\left\{x_{5,1}, x_{5,2}\right\}$; the cardinalities of the subsets are $\left|X_{1}\right|=$ $\left|X_{2}\right|=1,\left|X_{3}\right|=\left|X_{4}\right|=\left|X_{5}\right|=2$, indicating that $\boldsymbol{d}=(1,1,2,2,2)$. The multihomogeneous degree is $\delta_{\left(X_{i}, X_{j}\right)}=1$ for all $\left(X_{i}, X_{j}\right) \in E$ but, of course, there are some terms missing due to vanishing coefficients.

The vertices of $G(\boldsymbol{Q})$ are labeled by these subsets. The edge set $E$ is:

$\left\{\left(X_{1}, X_{2}\right),\left(X_{1}, X_{3}\right),\left(X_{1}, X_{5}\right),\left(X_{2}, X_{4}\right),\left(X_{2}, X_{5}\right),\left(X_{3}, X_{4}\right),\left(X_{3}, X_{5}\right),\left(X_{4}, X_{5}\right)\right\}$.

We count orientations such that the outdegrees of $X_{1}, X_{2}$ is $d_{1}=d_{2}=1$, while that of $X_{3}, X_{4}, X_{5}$ is $d_{3}=d_{4}=d_{5}=2$. Thus the $m$-Bézout number is the same as the number of the orientations namely 6 (See Fig. 4). In that case this bound is exact, since the number of roots is also 6 , and so is the BKK bound.

We have $d=2$, so $\alpha_{2}=24^{1 / 5}$ and $\beta_{2}=18^{-1 / 5}$. We have $n=5$ and $k=2$, indicating that the bound from Theorem 1 is $\left\lfloor 24 \cdot 18^{-1 / 5}\right\rfloor=13$.

In order to use the Brègman-Minc bound, one constructs a matrix with rows representing the variables and columns representing the equations (see [12] for details). The entry $\left(i^{\prime}, j\right)$ equals $\delta_{i, j}$ for all $x_{i^{\prime}} \in X_{i}$. The matrix is: 

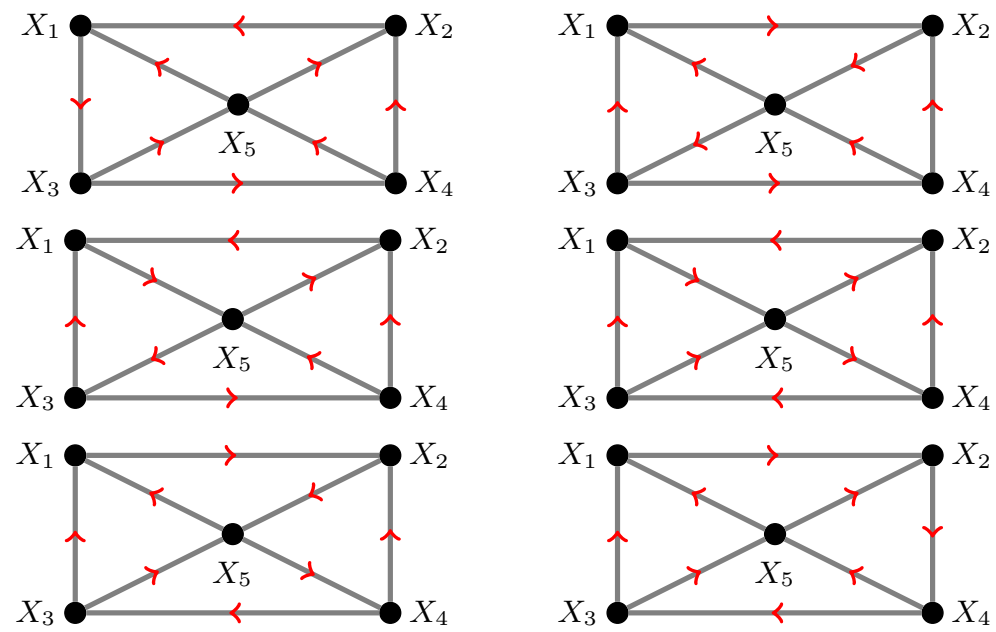

Fig. 4. The 6 valid orientations of the graph in Example 2

\begin{tabular}{c|llllllll} 
& $Q_{\left(X_{1}, X_{2}\right)}$ & $Q_{\left(X_{1}, X_{3}\right)}$ & $Q_{\left(X_{1}, X_{5}\right)}$ & $Q_{\left(X_{2}, X_{4}\right)}$ & $Q_{\left(X_{2}, X_{5}\right)}$ & $Q_{\left(X_{3}, X_{4}\right)}$ & $Q_{\left(X_{3}, X_{5}\right)}$ & $Q_{\left(X_{4}, X_{5}\right)}$ \\
\hline$x_{1,1}$ & 1 & 1 & 1 & 0 & 0 & 0 & 0 & 0 \\
$x_{2,1}$ & 1 & 0 & 0 & 1 & 1 & 0 & 0 & 0 \\
$x_{3,1}$ & 0 & 1 & 0 & 0 & 0 & 1 & 1 & 0 \\
$x_{3,2}$ & 0 & 1 & 0 & 0 & 0 & 1 & 1 & 0 \\
$x_{4,1}$ & 0 & 0 & 0 & 1 & 0 & 1 & 0 & 1 \\
$x_{4,2}$ & 0 & 0 & 0 & 1 & 0 & 1 & 0 & 1 \\
$x_{5,1}$ & 0 & 0 & 1 & 0 & 1 & 0 & 1 & 1 \\
$x_{5,2}$ & 0 & 0 & 1 & 0 & 1 & 0 & 1 & 1
\end{tabular}

The Brègman-Minc bound for $(0,1)$-matrices is $\prod_{i}\left(r_{i} !\right)^{1 / r_{i}}$, where $r_{i}$ is the sum of entries in row $i$. Thus the permanent is bounded by $6^{2} \cdot 24^{1 / 2}$. Based on Equation (3) one divides by $\prod_{i=1}^{n} d_{i} !=8$ and obtains a bound of $\lfloor 9 \sqrt{6}\rfloor=22$ on the m-Bézout number, which is looser than our method's.

The Bézout bound is 256, since all total degrees are 2 .

In both examples above, the maximum outdegree $d$ for a vertex in the associated graphs was 2 . To conclude let us give some brief examples for the computation of the bound using the closed formula of Theorem 1 for larger $d$, given the same graph with different cardinalities for the sets of variables. In all cases we will consider $\delta_{i}=1$.

The graph that will be analyzed has 6 vertices and 13 edges. The edge set is the following (see Fig. 5): $\left(X_{1}, X_{2}\right),\left(X_{1}, X_{3}\right),\left(X_{1}, X_{4}\right),\left(X_{1}, X_{5}\right),\left(X_{2}, X_{3}\right)$, $\left(X_{2}, X_{4}\right),\left(X_{2}, X_{5}\right),\left(X_{3}, X_{4}\right),\left(X_{4}, X_{5}\right),\left(X_{5}, X_{6}\right)$. 
We will first consider the case that the cardinalities are $\left|X_{1}\right|=\left|X_{3}\right|=1$, $\left|X_{4}\right|=2,\left|X_{2}\right|=|X 5|=\left|X_{6}\right|=3$. We have $d=3$, so $\alpha_{3}=40^{1 / 3}, \beta_{3}=200^{-1 / 9}$, while $k=5$. All these lead to $\left\lfloor 40^{2} \cdot 200^{-4 / 9}\right\rfloor=151$ as a bound.

If the cardinalities change so do the constraints on the outdegrees. For example for the following case $\left|X_{6}\right|=\left|X_{3}\right|=1,\left|X_{4}\right|=\left|X_{5}\right|=2,\left|X_{1}\right|=$ $3,\left|X_{2}\right|=4$ we have clearly that $d=4$, so $k=11$. This means that we shall use $\alpha_{4}=2^{9 / 13} \cdot 35^{5 / 13}, \beta_{4}=2^{-1 / 13} \cdot 35^{-2 / 13}$, concluding that the bound is $\left\lfloor 2^{43 / 13} \cdot 35^{8 / 13}\right\rfloor=160$.

Finally, let us present the case that $\left|X_{1}\right|=5,\left|X_{2}\right|=3,\left|X_{3}\right|=2,\left|X_{4}\right|=$ $\left|X_{5}\right|=\left|X_{6}\right|=1$. Now $d=5, k=17$ and also $\alpha_{5}=2^{19 / 17} \cdot 63^{7 / 17}$, $\beta_{5}=2^{-3 / 17} \cdot 63^{-2 / 17}$. The bound in that case is $\left\lfloor 2^{66 / 17} \cdot 63^{10 / 17}\right\rfloor=168$.

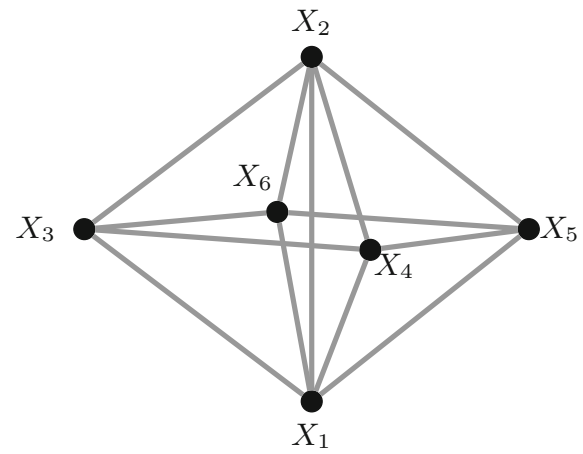

Fig. 5. .

\section{Conclusion}

In this paper, we studied methods that use the multi-homogeneous Bézout to improve the upper bounds on the number of embeddings of minimally rigid graphs. We generalized these methods to polynomial systems which represent simple graphs, and not only minimally rigid graphs. An open question is to further understand the algebraic implications of our results. The graph elimination process that yields the closed form bound on the number of orientations can be paralleled to algebraic variable elimination. The main open question is whether our approach may be extended to a wider class of well-constrained algebraic systems. This would require extending the proof that bounds the number of graph orientations to the graph corresponding to the more general class of algebraic systems.

Another open question is to obtain tight upper bound on the number of orientations of graphs. A result on this would immediately improve the upper bound on the m-Bézout number. This is actually our current work. A more theoretical question would be to estimate the error of our approximation. 


\section{References}

1. Baglivo, J., Graver, J.: Incidence and Symmetry in Design and Architecture. No. 7 in Cambridge Urban and Architectural Studies, Cambridge University Press (1983)

2. Bartzos, E., Emiris, I., Legerský, J., Tsigaridas, E.: On the maximal number of real embeddings of minimally rigid graphs in $\mathbb{R}^{2}, \mathbb{R}^{3}$ and $S^{2}$. J. Symbol. Comput. 102, 189-208 (2021). https://doi.org/10.1016/j.jsc.2019.10.015

3. Bartzos, E., Emiris, I., Schicho, J.: On the multihomogeneous Bézout bound on the number of embeddings of minimally rigid graphs. J. Appl. Algebra Eng. Commun. Comput. 31 (2020). https://doi.org/10.1007/s00200-020-00447-7

4. Bartzos, E., Emiris, I., Vidunas, R.: New upper bounds for the number of embeddings of minimally rigid graphs. arXiv:2010.10578 [math.CO] (2020)

5. Bernstein, D., Farnsworth, C., Rodriguez, J.: The algebraic matroid of the finite unit norm tight frame (FUNTF) variety. J. Pure Appl. Algebra 224(8) (2020). https://doi.org/10.1016/j.jpaa.2020.106351

6. Bernstein, D.: The number of roots of a system of equations. Func. Anal. Appl. 9(3), 183-185 (1975). https://doi.org/10.1007/BF01075595

7. Borcea, C., Streinu, I.: The number of embeddings of minimally rigid graphs. Discret. Comput. Geomet. 31(2), 287-303 (2004). https://doi.org/10.1007/s00454003-2902-0

8. Borcea, C., Streinu, I.: Periodic tilings and auxetic deployments. Math. Mech. Solids 26(2), 199-216 (2021). https://doi.org/10.1177/1081286520948116

9. Brègman, L.: Some properties of nonnegative matrices and their permanents. Dokl. Akad. Nauk SSSR 211(1), 27-30 (1973)

10. Cifuentes, D., Parrilo, P.: Exploiting chordal structure in polynomial ideals: a Gröbner bases approach. SIAM J. Discret. Math. 30(3), 1534-1570 (2016). https:// doi.org/10.1137/151002666

11. Emiris, I., Tsigaridas, E., Varvitsiotis, A.: Mixed volume and distance geometry techniques for counting Euclidean embeddings of rigid graphs. In: Mucherino, A., Lavor, C., Liberti, L., Maculan, N. (eds.) Distance Geometry: Theory, Methods and Applications, pp. 23-45. Springer, New York (2013). https://doi.org/10.1007/ 978-1-4614-5128-0_2

12. Emiris, I., Vidunas, R.: Root ounts of semi-mixed systems, and an application to counting Nash equilibria. In: Proceedings of ACM International Symposium Symbolic \& Algebraic Computation, pp. 154-161. ISSAC, ACM (2014). https:// doi.org/10.1145/2608628.2608679

13. Emmerich, D.: Structures Tendues et Autotendantes. Ecole d'Architecture de Paris, La Villette, France (1988)

14. Harris, J., Tu, L.: On symmetric and skew-symmetric determinantal varieties. Topology 23, 71-84 (1984)

15. Jackson, W., Owen, J.: Equivalent realisations of a rigid graph. Discrete Appl. Math. 256, 42-58 (2019). https://doi.org/10.1016/j.dam.2017.12.009. Special Issue on Distance Geometry: Theory \& Applications'16

16. Lavor, C., et al.: Minimal NMR distance information for rigidity of protein graphs. Discrete Appl. Math. 256, 91-104 (2019). www.sciencedirect.com/science/article/ pii/S0166218X18301793. Special Issue on Distance Geometry Theory \& Applications'16

17. Li, H., Xia, B., Zhang, H., Zheng, T.: Choosing the variable ordering for cylindrical algebraic decomposition via exploiting chordal structure. In: Proceedings of International Symposium on Symbolic and Algebraic Computation, ISSAC 2021. ACM (2021) 
18. Liberti, L.: Distance geometry and data science. TOP 28, 271-339 (2020)

19. Malajovich, G., Meer, K.: Computing minimal multi-homogeneous Bezout numbers is Hard. Theory Comput. Syst. 40(4), 553-570 (2007). https://doi.org/10.1007/ s00224-006-1322-y

20. Maxwell, J.: On the calculation of the equilibrium and stiffness of frames. Philos. Mag. 39(12) (1864)

21. Minc, H.: Upper bounds for permanents of $(0,1)$-matrices. Bull. AMS 69, 789-791 (1963). https://doi.org/10.1090/S0002-9904-1963-11031-9

22. Rocklin, D., Zhou, S., Sun, K., Mao, X.: Transformable topological mechanical metamaterials. Nat. Commun. 8 (2017). https://doi.org/10.1038/ncomms14201

23. Shafarevich, I.: Intersection Numbers, pp. 233-283. Springer, Heidelberg (2013). https://doi.org/10.1007/978-3-642-37956-7_4

24. Steffens, R., Theobald, T.: Mixed volume techniques for embeddings of Laman graphs. Comput. Geom. 43, 84-93 (2010)

25. Verschelde, J.: Modernizing PHCpack through phcpy. In: Proceedings of the 6th European Conference on Python in Science (EuroSciPy 2013), pp. 71-76 (2014)

26. Zelazo, D., Franchi, A., Allgöwer, F., Bülthoff, H.H., Giordano, P.: Rigidity maintenance control for multi-robot systems. In: Proceedings of Robotics: Science \& Systems, Sydney, Australia (2012)

27. Zhu, Z., So, A.C., Ye, Y.: Universal rigidity and edge sparsification for sensor network localization. SIAM J. Optim. 20(6), 3059-3081 (2010) 


\section{Author Queries}

Chapter 2

\begin{tabular}{|c|l|l|}
\hline $\begin{array}{l}\text { Query } \\
\text { Refs. }\end{array}$ & Details Required & $\begin{array}{l}\text { Author's } \\
\text { response }\end{array}$ \\
\hline AQ1 & $\begin{array}{l}\text { This is to inform you that corresponding author has been } \\
\text { identified as per the information available in the Copy- } \\
\text { right form. }\end{array}$ & \\
\hline AQ2 & Please provide the captions of Fig. 5, if available. & \\
\hline
\end{tabular}

